\title{
Improved outcomes from acute severe asthma in Australian intensive care units (1996-2003)
}

\section{Peter J Stow, David Pilcher, John Wilson, Carol George, Michael Bailey, Tracey Higlett, Rinaldo Bellomo, Graeme K Hart, and the Australian \& New Zealand Intensive Care Society Adult Patient Database Management Committee}

See end of article for authors' affiliations

Correspondence to: Dr David Pilcher, Intensive Care Unit, Alfred Hospital Commercial Road, Prahran 3181, Victoria, Australia; d.pilcher@alfred.org.au

Received 19 November 2006 Accepted 5 March 2007

Published Online First

27 March 2007
Background: There is limited information on changes in the epidemiology and outcome of patients with asthma admitted to intensive care units (ICUs) in the last decade. A database sampling intensive care activity in hospitals throughout Australia offers the opportunity to examine these changes.

Methods: The Australian and New Zealand Intensive Care Society Adult Patient Database was examined for all patients with asthma admitted to ICUs from 1996 to 2003. Demographic, physiological and outcome information was obtained and analysed from 22 hospitals which had submitted data continuously over this period.

Results: ICU admissions with the primary diagnosis of asthma represented $1899(1.5 \%)$ of 126906 admissions during the 8 -year period. $36.1 \%$ received mechanical ventilation during the first $24 \mathrm{~h}$. The overall incidence of admission to ICU fell from 1.9\% in 1996 to $1.1 \%$ in 2003 ( $p<0.001$ ). Overall hospital mortality was $3.2 \%$. There was a significant decline in mortality from a peak of $4.7 \%$ in 1997 to $1.1 \%$ in 2003 $(p=0.014)$. This was despite increasing severity of illness (as evidenced by an increasing predicted risk of death derived from the APACHE II score) over the 8-year period ( $p=0.002$ ).

Conclusions: There has been a significant decline in the incidence of asthma requiring ICU admission between 1996 and 2003 among units sampled by the Australian and New Zealand Intensive Care Society Adult Patient Database. The mortality of these patients has also decreased over time and is lower than reported in other studies.
A sthma severe enough to require admission to an intensive care unit (ICU) and the commencement of mechanical ventilation has generally only been studied in small centre-specific investigations. ${ }^{1-6}$ Divergent outcomes have been reported with significant variability in mortality. However, such studies have not addressed important wider epidemiological questions pertaining to trends in admission rates and the response to acute asthma treatment. Two reviews of sizeable populations extracted from high quality clinical databases have provided cross-sectional information on US and UK outcomes. ${ }^{78}$

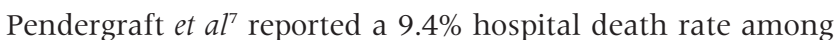
patients admitted to the ICU and requiring intubation in the year 2000. Gupta et al ${ }^{8}$ reported a hospital death rate of $15.4 \%$ in a similar group of patients treated in critical care units during 1995-2001. However, neither examined trends in the incidence of patients admitted to ICU or changes in mortality over time.

Studies from the USA ${ }^{9}$ and UK $^{10}$ have shown increasing mortality from asthma until the early 1990s, but a decline in more recent years. Australia is known to have had a higher asthma prevalence and mortality than other countries. ${ }^{11}$ Asthma deaths in Australia peaked in 1989 but had decreased by almost $30 \%$ in $2001 .^{12}$

The Australian and New Zealand Intensive Care Society (ANZICS) Adult Patient Database (APD) contains data from over 500000 adult admissions to 135 ICUs (from 1987). In 2004 there were 153 adult ICUs listed in Australia and New Zealand. By extracting Australian data only, we sought to test the hypotheses that:

- There has been a reduction in the number of patients with a primary diagnosis of asthma admitted to an ICU in Australia over an 8-year period (1996-2003).
- There has been a reduction in the number of patients requiring mechanical ventilation for asthma.

- There has been a reduction in hospital mortality for asthma over the 8-year period.

- The hospital mortality for such patients is $<5 \%$ and better than predicted by the APACHE II score.

\section{METHODS}

\section{Selection of patients and data}

We interrogated the ANZICS APD for all admissions with asthma during the period from 1 January 1996 to 31 December 2003. Only those Australian ICUs that had continuously contributed data over this period were selected. Paediatric admissions ( $<16$ years) were included if admitted to an adult ICU. The remaining data in the APD were analysed to determine whether outcomes observed in these 22 hospitals were representative of Australia.

ICU admissions with an APACHE II diagnostic coding of "asthma/allergy" were identified from the ANZICS APD. They were separated into those who required invasive mechanical ventilation within the first $24 \mathrm{~h}$ and those who did not. Demographic information including age, sex and date of presentation was extracted.

Abbreviations: ANZICS, Australian and New Zealand Intensive Care Society; APD, Adult Patient Database; COPD, chronic obstructive pulmonary disease; $\mathrm{FiO}_{2}$, fraction of inspired oxygen; ICU, intensive care unit; $\mathrm{PaO}_{2}, \mathrm{PaCO}_{2}$, arterial oxygen and carbon dioxide tensions 


\section{Severity of illness: APACHE II score, Glasgow coma score and physiological variables}

Severity of illness was assessed using the APACHE II score. ${ }^{13}$ From this, the predicted risk of death was calculated together with appropriate $95 \%$ confidence intervals (95\% CI) in accordance with the ANZICS APACHE II algorithm. Physiological (heart rate and blood pressure), biochemical (fraction of inspired oxygen $\left(\mathrm{FiO}_{2}\right)$, arterial oxygen and carbon dioxide tensions $\left(\mathrm{PaO}_{2}, \mathrm{PaCO}_{2}\right), \mathrm{pH}$, bicarbonate, urea, creatinine) and Glasgow coma score variables were extracted. In the APD, values are recorded to identify the most abnormal values within the first $24 \mathrm{~h}$ of admission to the ICU for severity scoring. Data about medication usage, smoking history and lung function tests are not recorded in the ANZICS APD.

\section{Outcomes}

Outcomes included the incidence of admission to the ICU (expressed as a proportion of total ICU admissions per year), the proportion of patients who required mechanical ventilation (instituted in the first $24 \mathrm{~h}$ ) and hospital mortality. These were examined annually and for the whole 8-year study period. When patients were admitted to the ICU on more than one occasion during a hospital stay, only the first ICU admission was included in the evaluation of mortality. The length of ICU stay (days) and discharge location (home, another hospital or rehabilitation facility) were also recorded.

\section{Statistical analysis}

Data were analysed using SAS V.8 (SAS Institute Inc, Cary, North Carolina, USA). Univariate comparisons were performed using the Student $t$ test, Wilcoxon rank sum test and $\chi^{2}$ test as appropriate. To determine changes over time, parametric and non-parametric tests for trend were performed. Parametric data are presented as mean (SD). Non-parametric data are expressed as median (interquartile range, IQR). All $p$ values $<0.05$ were considered significant. Multivariate models were constructed by a stepwise selection procedure and validated by a backward elimination procedure. All physiological and chronic health variables were used to build a multivariate model to determine factors associated with mortality. The same physiological values were used in the calculation of the APACHE II score. To avoid co-linearity, the APACHE II score was not included in the multivariate analysis.

\section{RESULTS}

\section{Demographic data}

Twenty two ICUs in Australia submitted data to the APD continuously over the 8-year period between 1996 and 2003. There were a total of 126906 adult admissions to these 22 units

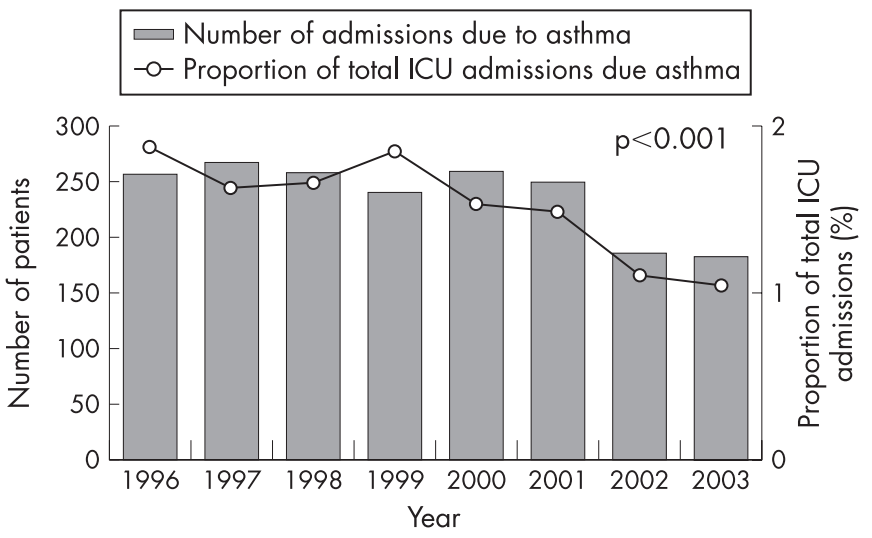

Figure 1 ICU admissions for asthma 1996-2003. and 1899 (1.5\%) had a primary diagnosis of asthma/allergy. A summary of the demographic data and outcomes is shown in table 1. The number of ICU admissions for asthma declined from a peak of 267 in 1997 to 183 in 2003 . When expressed as a proportion of overall ICU admissions, there was a significant decline from a peak of $1.9 \%$ in 1996 to $1.0 \%$ in 2003 ( $p<0.001$, fig 1).

\section{Mortality}

The hospital mortality for the 8-year cohort was 3.2\%. Over the 8 years there was a significant decline in mortality to a minimum of $1.1 \%(2 / 174)$ in $2003(p=0.014)$. During the later years, observed mortality fell below (in 2002) the lower $95 \%$ confidence interval for the APACHE II derived predicted mortality (fig 2). The factors significantly associated with mortality on multivariate analysis are shown in table 2 .

\section{Mechanical ventilation}

Mechanical ventilation was required in 685 of 1898 patients (36.1\%) during the first $24 \mathrm{~h}$. Although the absolute number declined over the 8 years, the percentage requiring ventilation remained unchanged (table 1). Mechanical ventilation was associated with mortality on multivariate analysis (OR 3.46, $95 \%$ CI $1.36 \%$ to $8.82 \%, p=0.009$; table 2 ). Of those requiring ventilation in the first $24 \mathrm{~h}, 6.5 \%$ died. This was similar to predicted mortality from the APACHE II score $(7.0 \%, 95 \%$ CI $6.3 \%$ to $7.6 \%)$. Patients who did not require ventilation in the first $24 \mathrm{~h}$ had lower mortality (1.3\%) than predicted by APACHE II $(2.4 \%, 95 \%$ CI $2.3 \%$ to $2.7 \%)$. There was a significant fall in mortality among ventilated patients over time to a minimum of $2.8 \%$ in $2002(\mathrm{p}=0.04$, fig 3$)$.

\section{Severity of illness: APACHE II score, Glasgow coma score and physiological variables}

The mean APACHE II predicted risk of death for the 8-year cohort was $3.9 \%$ (95\% CI $3.0 \%$ to $5.2 \%$ ) compared with the observed mortality of $3.2 \%$. There was a small but significant increase in APACHE II predicted risk of death $(\mathrm{p}=0.002)$ over the 8 -year period (fig 2 ) which peaked at $4.6 \%$ (95\% CI $1.3 \%$ to $36.6 \%)$ in 2002 .

Multivariate analysis confirmed that increasing Glasgow coma score was associated with a reduced likelihood of death (OR $0.89,95 \%$ CI 0.83 to $0.96, p=0.001$ ). Over the 8 -year period there was a reduction in the number of patients admitted with a Glasgow coma score of <6 (from 23 in 1996 and 1997 to 7 in 2003), but when expressed as a proportion of the total patients this was not statistically significant. However, in the group with a Glasgow coma score of $\geqslant 12$, there was a decrease in mortality from $3.8 \%$ in 1996 to $0 \%$ in 2003 $(\mathrm{p}=0.002)$.

Among patients who were ventilated in the first $24 \mathrm{~h}$, there was no difference in the $\mathrm{pH}$ values between survivors and nonsurvivors (mean (SD) $7.19(0.16)$ and $7.17(0.14)$, respectively; $\mathrm{p}=0.18)$. Similarly, there were no differences in the mean (SD) $\mathrm{PaCO}_{2}$ values between survivors and non-survivors (9.29 (4.40) $\mathrm{kPa}$ and 9.88 (3.88) $\mathrm{kPa}$, respectively). However, among those who were not ventilated in the first $24 \mathrm{~h}$, non-survivors had lower $\mathrm{pH}$ values $(7.23(0.15)$ and $7.34(0.11) ; \mathrm{p}=0.02)$ and higher $\mathrm{PaCO}_{2}$ values (10.39 (3.36) kPa and 6.00 (2.87) kPa; $\mathrm{p}<0.001$ ) than survivors. In multivariate analysis (table 2 ), the only respiratory physiological variable associated with mortality was pH (OR 0.10, 95\% CI 0.01 to 0.99 ; p = 0.048).

Physiological variables are shown in tables 3 and 4 .

\section{Validation of the dataset}

The APD is known to offer a representative picture of admission and outcome patterns for the intensive care population in 
Table 1 Summary of demographic data and outcomes for patients admitted to an ICU with asthma/allergy, 19962003

\begin{tabular}{ll}
\hline Total number of admissions to ICU & 1899 \\
Total number of patients (after exclusion of & \\
readmissions) & 1858 \\
Mean (SD) age (years) & $39.6(18.4)$ \\
Proportion of admissions $\leqslant 16$ years of age & $7.9 \%(150 / 1899)$ \\
Proportion of admissions $>45$ years of age & $35.5 \%(675 / 1899)$ \\
Proportion female & $64.8 \%(1229 / 1899)$ \\
Proportion of admissions listed as having & $16.9 \%(314 / 1858)$ \\
comorbid chronic respiratory conditions* & \\
Median (IQR) duration of ICU stay (h) & $38(18.7-72.0)$ \\
Proportion ventilated in first 24 $\mathrm{h}$ & $36.1 \%(685 / 1898)$ \\
Median (SD) predicted risk of death from & $3.9 \%(5.6)$ \\
APACHE II score & $3.20 \%(60 / 1866)$ \\
Observed mortality &
\end{tabular}

$I C U$, intensive care unit; $I Q R$, interquartile range; $S D$, standard deviation. ${ }^{*}$ Chronic respiratory disease is defined as chronic restrictive, obstructive or vascular respiratory disease resulting in severe exercise restriction, ie, unable to climb stairs or perform household duties; or documented chronic hypoxia, hypercapnia, secondary polycythaemia, severe pulmonary hypertension (> $40 \mathrm{~mm} \mathrm{Hg}$ ) or respiratory dependency.

Australia. ${ }^{14}$ Of 135 hospital ICUs which submitted data during this 8-year period, only 22 did so continuously. More hospitals were not included to analyse trends over time because gaps in data submission might have been interpreted as variation in incidence. However, we analysed the overall demographics of the remainder of the database. This listed 232381 admissions to intensive care between 1996 and 2003, of which 3578 (1.5\%) had a primary diagnosis of asthma/allergy. The mortality of these patients was $3.1 \%$. These admission and death rates were the same as for the 22 hospitals in our main analysis $(p=0.34$ and $p=0.93$, respectively). All eight states and territories of Australia were represented by these 22 hospitals and all types of hospital were represented ( 3 rural, 6 metropolitan, 9 tertiary and 4 private hospitals).

\section{DISCUSSION}

The main findings of our study are that, over an 8-year period, there has been a reduction in the absolute number and proportion of patients admitted to ICU with asthma, that the proportion of ICU admissions who require mechanical ventilation in the first $24 \mathrm{~h}$ has remained unchanged, and that the mortality of these patients has significantly declined, despite increasing severity of illness.

Guidelines for the management of asthma were first produced by the Thoracic Society of Australia and New Zealand in 1989 at a time of peak mortality from asthma. Written asthma management plans ${ }^{15}$ and inhaled preventer medications $^{16}$ are known to reduce admissions to hospital. Improvement in management of asthma in the community has reduced hospital admissions (reported in Australia ${ }^{11}$ and other countrie ${ }^{17}$ ) and has led to a reduction in ICU admissions.

Although the proportion of patients requiring mechanical ventilation (expressed as a percentage of all ICU admissions for asthma) remained constant over the study period, there was a reduction in the absolute numbers of patients. A similar trend has recently been reported by the Australian Centre for Asthma Monitoring (data from the Australian National Hospital Morbidity Database). ${ }^{11}$ We report $36.1 \%$ of patients requiring mechanical ventilation in the first $24 \mathrm{~h}$. However, it is likely that the overall requirement for mechanical ventilation was higher than this as mechanical ventilation instituted after the first day is not recorded in the ANZICS APD. Rates of mechanical ventilation between $15.4 \%$ in the USA ${ }^{7}$ and $57 \%$

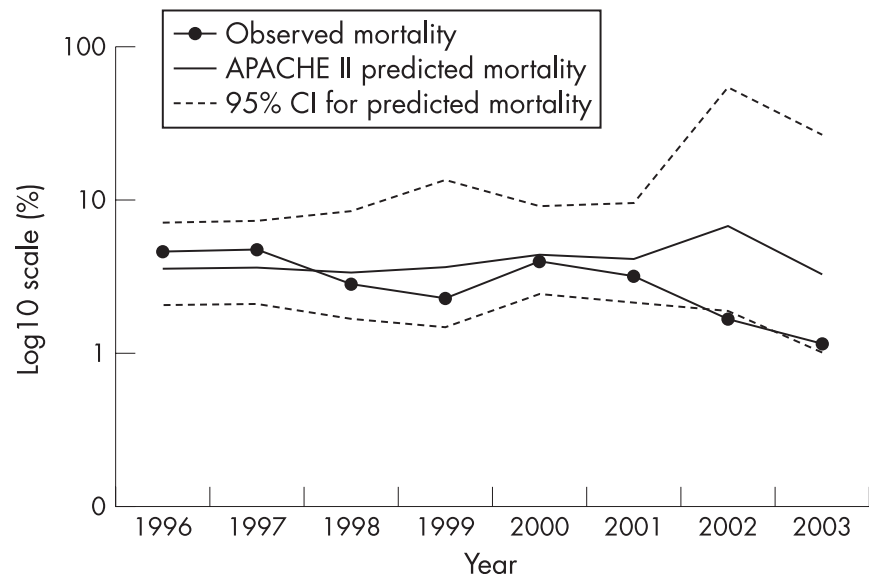

Figure 2 Observed mortality compared with predicted mortality (using APACHE II score).

in the $\mathrm{UK}^{8}$ have been reported, which may reflect differing ICU admission policies and ICU bed availability.

We found an overall hospital death rate of 3.2\%. Although this was below the APACHE II derived predicted risk of death, this value fell between the $95 \%$ confidence intervals $13.0 \%$ to $5.2 \%$ ). Thus, our observed mortality cannot be considered to be significantly better than predicted by APACHE II, a scoring system previously validated for the assessment of severity of illness in ICU admissions with asthma. ${ }^{18}$

We have shown that the mortality from severe asthma requiring admission to ICUs sampled in Australia has decreased significantly over an 8-year period for all patients and for those requiring mechanical ventilation. Declining mortality has recently been described in Australia, ${ }^{11}$ the UK, ${ }^{10}$ South Africa ${ }^{19}$ and the USA. ${ }^{90}$ In 1990 the mortality associated with asthma in Australia was 5.6 per 100000 population; by 2003 it had declined to 1.5 per 100000 population. ${ }^{11}$ It is therefore unlikely that the reduction in ICU deaths observed in our study was due to an excess of deaths in the general community outside hospital or delayed access to specialist care facilities. Furthermore, the reduction in deaths among patients admitted to ICUs was not because a greater proportion of those admitted were "less sick". The disease severity of patients admitted to the ICU (as estimated by the APACHE II score) increased over time; this implies improved patient management in the ICU.

Many factors may have combined to lead to the improved outcomes seen in our study, but it was neither designed nor powered to investigate which treatments may have caused this. Factors such as intravenous magnesium, ${ }^{21}$ leucotriene antagonists, ${ }^{22}$ non-invasive ventilation (known to be beneficial in exacerbations of chronic obstructive pulmonary disease

Table 2 Multivariate analysis for factors associated with mortality

\begin{tabular}{lll}
\hline & Odds ratio $(95 \% \mathrm{Cl})$ & $\mathrm{p}$ Value \\
\hline Age & $1.04(1.02$ to 1.06$)$ & $<0.0001$ \\
Urea & $1.06(1.03$ to 1.10$)$ & $<0.001$ \\
Glasgow coma score & $0.89(0.83$ to 0.96$)$ & 0.001 \\
Immunosuppression* & $5.04(1.64$ to 15.49$)$ & 0.005 \\
Mechanical ventilation & $3.46(1.36$ to 8.82$)$ & 0.009 \\
Mean blood pressure & $0.98(0.97$ to 0.99$)$ & 0.012 \\
Arterial pH & $0.10(0.01$ to 0.99$)$ & 0.048 \\
\hline
\end{tabular}

*A patient was coded as having immunosuppression if there was either disease sufficiently advanced to suppress resistance to infection (eg, leukaemia, lymphoma, AIDS) or if he/she had received treatment that suppresses resistance to infection (eg, immunosuppressive drugs, chemotherapy, radiation, long-term or recent high-dose steroids). 


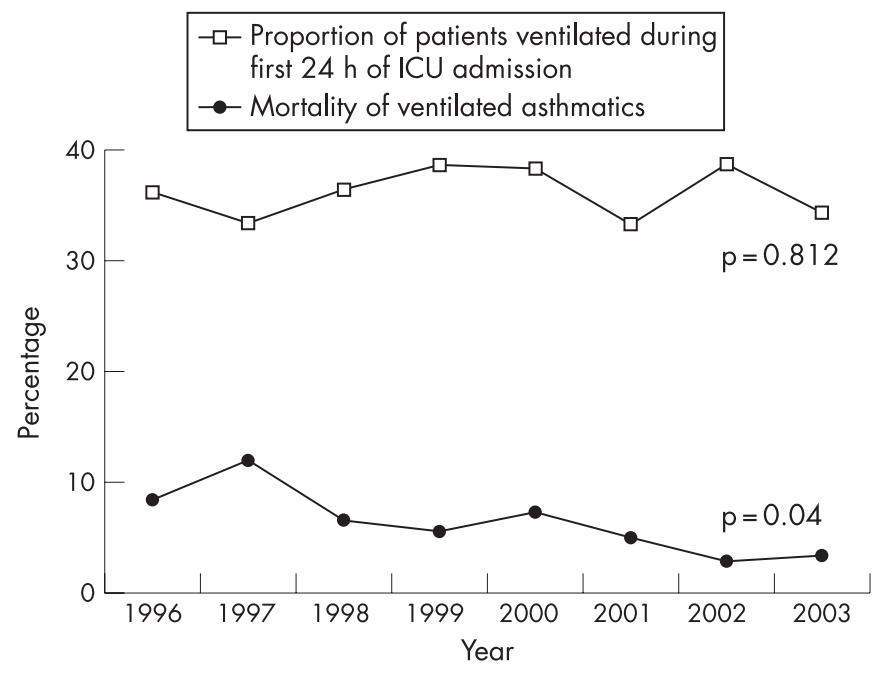

Figure 3 Incidence of ventilation and mortality of ventilated patients admitted to an intensive care unit (ICU) with asthma.

$\left.(\mathrm{COPD})^{23}\right)$, and possibly increased awareness of detrimental effects associated with positive pressure ventilation in patients with asthma ${ }^{24-26}$ may have contributed to improved outcomes.

Early identification and intervention in a deteriorating patient (either on the ward to prompt ICU admission or within the ICU to prompt initiation of rescue therapies) is also likely to have been a factor. This is supported by the finding that the mortality of those who were not ventilated in the first $24 \mathrm{~h}$ $(1.3 \%)$ is below the $95 \%$ confidence interval for the predicted mortality from the APACHE II score. The importance of the appropriate intervention in these patients is significant since non-survivors who were not ventilated in the first $24 \mathrm{~h}$ had a lower $\mathrm{pH}$ and a higher $\mathrm{PaCO}_{2}$ level than those who did survive. It is unknown whether earlier consideration of mechanical ventilation in these patients might have improved outcomes even further.

The hospital mortality in our cohort of patients $(3.2 \%)$ is similar to that reported by McFadden ${ }^{27}$ but lower than that reported by Gupta et $\mathrm{al}^{8}(9.8 \%)$ and Pendergraft et $\mathrm{al}^{7}(9.4 \%)$. We observed a higher mortality $(6.5 \%)$ among patients requiring mechanical ventilation. Similar trends were seen in the studies by McFadden ${ }^{27}(8.1 \%)$ and Gupta et $a^{8}(15.4 \%)$. Multivariate analysis confirmed the significance of the association between mechanical ventilation and mortality.

Multivariate analysis showed that a number of "nonrespiratory" factors such as increasing age, rising urea, reduced Glasgow coma score, immunosuppression and reduced blood pressure were associated with mortality. This implies that derangement in other physiological systems, either due to preexisting disease or secondary to the severity of their asthma (eg, following cardiac arrest), were the most important in determining survival. Indeed, co-morbidities including obesity ${ }^{28}$ and depression ${ }^{29}$ are already known to contribute to poor asthma control and suboptimal outcome from acute exacerbations. However, it is unknown whether the association between immunosuppression and mortality implies mortality directly attributable to high-dose steroids (or drugs such as methotrexate and cyclosporine) or whether this simply reflects that patients who have the "most difficult to control" asthma (ie, most likely to be taking immunosuppressant medications) are those most likely to die. Of the 77 admissions who were coded as being immunosuppressed, $62 \%$ were on immunosuppressive treatment. The remainder had immunosuppressive diseases which might also have contributed directly to the mortality risk.

The major strength of our study is that accurately recorded data and a large study population allow close examination of

Table 3 Univariate comparison of patients ventilated within the first $24 \mathrm{~h}$ with those not ventilated within the first $24 \mathrm{~h}$

\begin{tabular}{|c|c|c|c|}
\hline & \multicolumn{2}{|c|}{ Mechanical ventilation in first $24 \mathrm{~h}$} & \multirow[b]{2}{*}{$\mathrm{p}$ Value } \\
\hline & Yes $(n=685)$ & No $(n=1213)$ & \\
\hline \multicolumn{4}{|l|}{ Outcomes } \\
\hline Mortality, \% (n) & $6.5 \%(43 / 660)$ & $1.3 \%(14 / 1165)$ & $<0.001$ \\
\hline Duration of ICU stay (days) & $2.6(1.4-5.9)$ & $1.2(0.7-2.1)$ & $<0.001$ \\
\hline \multicolumn{4}{|l|}{ Demographic data } \\
\hline Predicted mortality from APACHE II & $7.0 \%(95 \% \mathrm{Cl} 6.3 \%$ to $7.6 \%)$ & $2.4 \%(95 \% \mathrm{Cl} 2.3 \%$ to $2.7 \%)$ & $<0.001$ \\
\hline Age (years) & $43.6(18.3)$ & $37.5(18.1)$ & $<0.001$ \\
\hline Proportion $\leqslant 16$ years & $4.7 \%(32 / 681)$ & $9.7 \%(118 / 1211)$ & $<0.001$ \\
\hline Proportion $>45$ years & $45.5 \%(312 / 685)$ & $29.9 \%(363 / 1213)$ & $<0.001$ \\
\hline $\begin{array}{l}\text { Proportion with chronic respiratory } \\
\text { disease }^{*}\end{array}$ & $16.8 \%(129 / 685)$ & $16.8 \%(204 / 1213)$ & 0.998 \\
\hline Proportion female & $64 \%(438 / 685)$ & $65 \%(791 / 1213)$ & 0.562 \\
\hline \multicolumn{4}{|l|}{ Physiology } \\
\hline Pulse & $132(24)$ & $132(23)$ & 0.927 \\
\hline Mean arterial blood pressure $(\mathrm{mm} \mathrm{Hg})$ & $86(1.1)$ & $87(0.6)$ & 0.253 \\
\hline $\mathrm{pH}$ from $\mathrm{ABG}$ with lowest $\mathrm{PaO}_{2}$ & $7.18(0.15)$ & $7.34(0.11)$ & $<0.001$ \\
\hline $\mathrm{PaCO}_{2}$ from $\mathrm{ABG}$ with lowest $\mathrm{pH}(\mathrm{kPa})$ & $9.32(4.36)$ & $6.07(2.91)$ & $<0.001$ \\
\hline Bicarbonate (mmol) & $24.0(6.5)$ & $22.3(5.9)$ & $<0.001$ \\
\hline Lowest $\mathrm{PaO}_{2}(\mathrm{kPa})$ & $22.13(15.87)$ & $15.73(9.33)$ & $<0.001$ \\
\hline $\mathrm{PaO}_{2} / \mathrm{FiO}_{2}$ ratio & $277(171)$ & $275(148)$ & 0.844 \\
\hline Glasgow coma score & $12.2(4.5)$ & $14.7(1.5)$ & $<0.001$ \\
\hline Urea $(\mathrm{mmol} / \mathrm{l})$ & $6.60(0.22)$ & $6.28(0.26)$ & 0.401 \\
\hline Creatinine $(\mu \mathrm{mol} / \mathrm{l})$ & $94.3(2.6)$ & $78.9(0.9)$ & $<0.001$ \\
\hline
\end{tabular}

$I C U$, intensive care unit; $A B G$, arterial blood gas; $\mathrm{PaO}_{2}, \mathrm{PaCO}_{2}$, arterial oxygen and carbon dioxide tensions; $\mathrm{FiO}_{2}$, fraction of inspired oxygen.

All values reported as mean (SD) except length of stay reported as median (IQR).

All values recorded are the worst within the first $24 \mathrm{~h}$.

${ }^{*}$ Chronic respiratory disease is defined as chronic restrictive, obstructive or vascular respiratory disease resulting in severe exercise restriction, ie, unable to climb stairs or perform household duties; or documented chronic hypoxia, hypercapnia, secondary polycythaemia, severe pulmonary hypertension (>40 mm Hg) or respiratory dependency. 
Table 4 Univariate comparison of survivors and non-survivors

\begin{tabular}{|c|c|c|c|}
\hline & \multicolumn{2}{|c|}{ Hospital survival (readmissions to ICU excluded) } & \multirow[b]{2}{*}{$\mathrm{p}$ Value } \\
\hline & Yes $(n=1768)$ & No $(n=58)$ & \\
\hline \multicolumn{4}{|l|}{ Outcomes } \\
\hline Duration of ICU stay (days) & $1.6(0.7-2.9)$ & $4.6(1.4-9.2)$ & $<0.001$ \\
\hline Proportion ventilated in first $24 \mathrm{~h}$ & $34.9 \%(617 / 1768)$ & $74.1 \%(43 / 58)$ & $<0.001$ \\
\hline \multicolumn{4}{|l|}{ Demographic data } \\
\hline Age (years) & $38.9(18.1)$ & $57.2(19.3)$ & $<0.001$ \\
\hline Proportion $\leqslant 16$ years & $8.4 \%(148 / 1768)$ & $1.7 \%(1 / 58)$ & 0.251 \\
\hline Proportion $>45$ years & $34.2 \%(605 / 1768)$ & $72.4 \%(42 / 58)$ & $<0.001$ \\
\hline Proportion with chronic respiratory disease* & $16.0 \%(283 / 1768)$ & $32.7 \%(19 / 58)$ & $<0.001$ \\
\hline Proportion female & $64.5 \%(628 / 1768)$ & $65 \%(37 / 58)$ & 0.887 \\
\hline \multicolumn{4}{|l|}{ Physiology } \\
\hline Pulse & $132(23)$ & $132(27)$ & 0.956 \\
\hline Mean arterial blood pressure $(\mathrm{mm} \mathrm{Hg})$ & $87(0.6)$ & $79(3.7)$ & $<0.001$ \\
\hline $\mathrm{pH}$ from $\mathrm{ABG}$ with lowest $\mathrm{PaO}_{2}$ & $7.28(0.15)$ & $7.17(0.14)$ & $<0.001$ \\
\hline $\mathrm{PaCO}_{2}$ from $\mathrm{ABG}$ with lowest $\mathrm{pH}(\mathrm{kPa})$ & $7.24(3.87)$ & $9.88(0.02)$ & $<0.001$ \\
\hline Bicarbonate (mmol) & $22.9(6.0)$ & $25.1(8.2)$ & 0.009 \\
\hline $\mathrm{PaO}_{2}(\mathrm{kPa})$ & $17.33(12.67)$ & $19.60(16.00)$ & 0.412 \\
\hline $\mathrm{PaO}_{2} / \mathrm{FiO}_{2}$ ratio & 277 (159) & $241(161)$ & 0.099 \\
\hline Glasgow coma score & $13.9(2.9)$ & $10.5(5.1)$ & $<0.001$ \\
\hline Urea (mmol/l) & $6.2(0.18)$ & $10.46(1.3)$ & $<0.001$ \\
\hline Creatinine $(\mu \mathrm{mol} / \mathrm{l})$ & $83.3(1.1)$ & $126.1(11.9)$ & $<0.001$ \\
\hline \multicolumn{4}{|c|}{$\begin{array}{l}\text { ICU, intensive care unit; } \mathrm{ABG} \text {, arterial blood gas; } \mathrm{PaO}_{2}, \mathrm{PaCO}_{2} \text {, arterial oxygen and carbon dioxide tensions; } \mathrm{FiO}_{2} \text {, } \\
\text { fraction of inspired oxygen. } \\
\text { All values reported as mean (SD) except length of stay reported as median (IQR). } \\
\text { All values recorded are the worst within the first } 24 \mathrm{~h} \text {. } \\
\text { *Chronic respiratory disease is defined as chronic restrictive, obstructive or vascular respiratory disease resulting in } \\
\text { severe exercise restriction, ie, unable to climb stairs or perform household duties; or documented chronic hypoxia, } \\
\text { hypercapnia, secondary polycythaemia, severe pulmonary hypertension ( }>40 \mathrm{~mm} \mathrm{Hg} \text { ) or respiratory dependency. } \\
32 \text { patients had no outcome recorded, } 41 \text { patients had readmissions to ICU. }\end{array}$} \\
\hline
\end{tabular}

changes over time. The ANZICS APD is the world's largest repository of intensive care data and is representative of Australian intensive care practice. ${ }^{14}$ The overall incidence and mortality for ICU admissions with asthma were the same in the 22 studied hospitals as in the remaining submissions to the APD. Our findings are therefore likely to be representative of asthma outcomes throughout Australia and New Zealand.

There are potential limitations to our study. Selecting an APACHE II diagnosis of asthma/allergy may have introduced bias. Some of the patients included may have had an allergic or anaphylactic reaction without asthma. APACHE II is the most widely reported and used system for scoring "severity of illness". It is the only system that has been specifically validated to assess disease severity in acute asthma. ${ }^{18}$ There are a number of other severity of illness scoring systems. The ANZICS APD has more recently collected data for the APACHE III scoring system which has a separate subcode for asthma; 1629 of $1899(86 \%)$ patients in our cohort had an APACHE III subcode of "asthma". We can therefore be confident that these were admissions solely due to asthma. The remaining 270 admissions did not have data collected for APACHE III.

Patients with COPD may have been included in the cohort. $16.9 \%$ of the admissions were coded as having chronic respiratory conditions (which may or may not have been COPD) in addition to the primary admission diagnosis of asthma. Approximately one third of admissions were in patients aged $>45$ years of age, of whom an unknown percentage may have had COPD. This is a problem common to other studies of asthma. Patients in the study by Gupta et $a l^{8}$ were of similar age (mean (SD) 43.6 (19.2) years) to those in our study. Indeed, over $50 \%$ of the patients in the study by Pendergraft et al were aged $>40$ years. Furthermore, it is also possible that our study may have missed a small number of patients with asthma. For example, patients with severe asthma who suffered a pre-hospital cardiac arrest may have been coded as cardiac arrest and not asthma. Finally, as this is not a controlled study, we are unable to describe with certainty the aspects of care responsible for the improved outcomes observed.

In conclusion, the incidence of asthma in Australia requiring ICU admission is low and has decreased significantly from 1996 to 2003. The mortality of patients with asthma requiring ICU admission has also decreased significantly from 1996 to 2003, despite increasing severity of illness among those admitted. Finally, the mortality due to asthma in patients requiring ICU admission in Australia is now lower than that reported in similar studies.

\section{Authors' affiliations}

Peter J Stow, Department of Intensive Care, Geelong Hospital, Geelong, Australia

David Pilcher, Department of Intensive Care, Alfred Hospital, Melbourne, Australia

John Wilson, Department of Allergy, Immunology and Respiratory Medicine, Alfred Hospital, Melbourne, Australia

Peter J Stow, David Pilcher, Carol George, Rinaldo Bellomo, Graeme

K Hart, Australia and New Zealand Intensive Care Society (ANZICS) Adult Patient Database, Melbourne, Australia

Michael Bailey, Department of Epidemiology and Preventive Medicine,

Monash University, Prahran, Australia

Tracey Higlett, ANZICS Research Centre for Critical Care Resources,

Melbourne, Australia

Rinaldo Bellomo, Graeme K Hart, Department of Intensive Care, Austin

Hospital, Melbourne, Australia

Funding: None.

Competing interests: None.

\section{REFERENCES}

1 Afessa B, Morales I, Cury J. Clinical course and outcome of patients admitted to an ICU for status asthmaticus. Chest 2001;120:1616-21.

2 Darioli R, Perret C. Mechanical controlled hypoventilation in status asthmaticus. Am Rev Respir Dis 1984;129:385-7. 
3 Gehlbach B, Kress J, Kahn J, et al. Correlates of prolonged hospitalisation in inner-city ICU patients receiving non-invasive and invasive positive pressure ventilation for status asthmaticus. Chest 2002;122:1709-14.

4 Higgins B, Greening A, Crompton G. Assisted ventilation in severe acute asthma. Thorax 1986:41:464-7.

5 Luksza A, Smith P, Coakley J, et al. Acute severe asthma treated by mechanical ventilation: 10 years' experience from a district general hospital. Thorax 1986;41:459-63.

6 Picado C, Montserrat J, Roca J, et al. Mechanical ventilation in severe exacerbation of asthma. Study of 26 cases with six deaths. Eur J Respir Dis 1983;64:102-7.

7 Pendergraft T, Stanford R, Beasley R, et al. Rates and characteristics of intensive care unit admissions and intubations among asthma-related hospitalisations. Ann Allergy Asthma Immunol 2004;93:29-35.

8 Gupta D, Keogh B, Chung K, et al. Characteristics and outcome for admissions to adult, general critical care units with acute severe asthma: a secondary analysis of the ICNARC Case Mix Programme Database. Crit Care 2004;8:R112-21.

9 Mannino D, Homa D, Akinbami L, et al. Surveillance for asthma: United States, 1980-1999. MMWR Surveill Summ 2002;51:1-13.

10 Campbell M, Cogman G, Holgate S, et al. Age specific trends in asthma mortality in England and Wales, 1983-95: results of an observational study BMJ 1997;314:1439.

11 Australian Centre for Asthma Monitoring. Asthma in Australia 2005, AlHW Asthma Series 2. Canberra: AlHW, 2005.

12 Woolcock A, Bastiampillai S, Marks G, et al. The burden of asthma in Australia. Med J Aust 2001;175:141-5.

13 Knaus W, Draper E, Wagner D, et al. APACHE II: a severity of disease classification system. Crit Care Med 1985;13:818-29.

14 Duke G, Santamaria J, Shann F, et al. Outcome-based clinical indicators for intensive care medicine. Anaesth Intensive Care 2005;33:303-10.

15 Gibson P, Powell H, Coughlan J, et al. Self-management education and regular practitioner review for adults with asthma. In: Cochrane Library, Issue 3. Oxford: Update Software, 2002.

16 Suissa S, Ernst P. Inhaled corticosteroids: impact on asthma morbidity and mortality. J Allergy Clin Immunol 2001;107:937-44.
17 Vargas M, Guillermo S-M, Furuya M, et al. Trends of asthma in Mexico: an 11 year analysis in a nationwide institution. Chest 2004;125:1993-7.

18 Day A, Rankin A, Judson J. Grading asthma severity: using the APS component of the APACHE II system. Intensive Care Med 1993;19:221-6.

19 Zar H, Stickells D, Toerien A, et al. Changes in fatal and near-fatal asthma in an urban area of South Africa from 1980-1997. Eur Respir J 2001;18:33-7.

20 Sly R. Continuing decreases in asthma mortality in the United States. Ann Allergy Asthma Immunol 2004:92:313-8.

21 Silverman R, Osborn $\mathrm{H}$, Runge J, et al. Intravenous magnesium sulphate in the treatment of acute severe asthma: a multicentre randomised controlled trial. Chest 2002;122:489-97.

22 Silverman RA, Nowak RM, Korenblat PE, et al. Zafirlukast treatment for acute asthma: evaluation in a randomized, double-blind, multicenter trial. Chest 2004;126:1480-9.

23 Plant PK, Owen JL, Elliott MW. Early use of non-invasive ventilation for acute exacerbations of chronic obstructive pulmonary disease on general respiratory wards: a multicentre randomised controlled trial. Lancet 2000;355:1931-5.

24 Bellomo R, McLaughlin P, Tai E, et al. Asthma requiring mechanical ventilation. A low morbidity approach. Chest 1994;105:891-6.

25 Williams T, Tuxen D, Scheinkestel C, et al. Risk factors for morbidity in mechanically ventilated patients with acute asthma. Am Rev Respir Dis 1992;146:607-15.

26 Tuxen DV, Lane S. The effects of ventilatory pattern on hyperinflation, airway pressures, and circulation in mechanical ventilation of patients with severe airflow obstruction. Am Rev Respir Dis 1987;136:872-9.

27 McFadden E. Acute severe asthma. Am J Respir Crit Care Med 2003;168:740-59.

28 Carroll CL, Bhandari A, Zucker AR, et al. Childhood obesity increases duration of therapy during severe asthma exacerbations. Pediatr Crit Care Med 2006; 7:527-31.

29 Dahlen I, Janson C. Anxiety and depression are related to the outcome of emergency treatment in patients with obstructive pulmonary disease. Chest 2002;122:1633-7.

\section{LUNG ALERT}

\section{Microscopic observation drug susceptibility assay for the diagnosis of TB}

$\Delta$ Moore DAJ, Evans CAW, Gilman RH, et al. Microscopic observation drug susceptibility assay for the diagnosis of TB. N Engl $J$ Med 2006;355:1539-50.

$E^{2}$ xisting strategies to control the spread of tuberculosis (TB) miss important opportunities to interrupt transmission. This study compares the microscopic observation drug susceptibility (MODS) assay with conventional reference methods of automated mycobacterium culture or culture on Lowenstein-Jenson medium.

This study, conducted in Peru in 2003-2004, targeted three patient groups: unselected patients with suspected TB, pre-screened patients at high risk of TB or multidrug-resistant (MDR) TB and unselected hospitalised patients infected with HIV. From 10 government clinics and two hospitals 1980 patients were recruited; 3760 sputum samples were collected, out of which 401 $(10.7 \%)$ yielded culture positive results for MDR compared with 220 (5.9\%) sputum smear positive results. The sensitivity of detection using MODS assay, automated mycobacterium culture and Lowenstein-Jenson medium culture was $97.8 \%, 89 \%$ and $84 \%$, respectively. The median time to culture positivity was 7 days for MODS assay, 13 days for automated mycobacterium culture and 26 days for Lowenstein-Jenson medium. The median time to results of susceptibility tests was 7 days for MODS assay compared with 22 days for automated mycobacterium culture and 68 days for Lowenstein-Jenson medium. Agreement between MODS assay and reference standard for susceptibility was $100 \%$ for rifampicin, $96.7 \%$ for isoniazid, $98.8 \%$ for combination of rifampicin and isoniazid, $95.4 \%$ for ethambutol and $91.7 \%$ for streptomycin.

The study was designed to include broader groups of patients with or without disease and in pertinent patient groups. The authors concluded that MODS assay detected mycobacterium TB in sputum with greater sensitivity and speed and reliably identified MDR TB in less time than conventional reference methods. However, the study did not comment on application of this assay in clinical scenarios and the role of this assay in the future.

This intriguing study hints to future developments in the diagnosis of TB and application of the MODS assay in normal day practice.

Talha Munir

Senior House Officer, Barnet Hospital, UK; munirtalha@aol.com 